\title{
PERFORMANCE ANALYSIS OF IEEE 802.15.4/ZIGBEE STANDARD UNDER REAL TIME CONSTRAINTS
}

\author{
Lamia CHAARI and Lotfi KAMOUN \\ SFAX University, National Engineering School (ENIS), TUNISIA \\ lamia.chaarietunet.tn
}

\begin{abstract}
In recent years, very interesting researches are concentrated to develop several protocols for Wireless sensor networks (WSN) and to explore WSN in various scenarios. The standardization of the IEEE 802.15.4 protocol as a flexible communication solution for low-rate wireless sensor networks leads to the widespread deployment of this technology in many potential applications with different requirements especially in industrial automation. The IEEE 802.15.4 specifies physical and media access control layers that could be optimized to ensure low-power consumption and to guarantee real-time constraints by using its guaranteed time slot (GTS) mechanism and enabling Beacon mode. Our focus in this paper is on real-time capabilities and reliability. We analyzed and compared the performance of the IEEE 802.15.4 standard using OMNET++ Simulator. Among the objectives of our study is to outline which degree the standard accomplish real time requirements. Different application scenarios were been evaluated. The analysis gives a full understanding of the behavior of the IEEE 802.15 .4 access mechanism concerning data delivery delay, goodput, throughput and packet errors rate metrics. We have focused on single sink scenarios and we have analyzed some network performance according to nodes number. Our simulation results pave the way for an efficient dimensioning and deploying of an IEEE 802.15.4 cluster.
\end{abstract}

\section{KEYWORDS}

IEEE 802.15.4; Zigbee; GTS, Medium Access control, OMNET++; Real time, Simulation.

\section{INTRODUCTION}

Recent development of communication technology has facilitated the expansion of the wireless sensors networks (WSN) [1][2][3]. The applicable area of WSN includes military sensing, data broadcasting [4], environmental monitoring [6], Intelligent Vehicular Systems [7], multimedia [8], patient monitoring [9], agriculture [10] [11], industrial automation [12] [13] [14] and audio [15] etc. WSN networks have not yet achieved widespread deployments, although they have been proven capable to meet the requirements of many applications categories. WSN has some limitations as lower computing power, smaller storage devices, narrower network bandwidth and very lower battery power. Real-time applications of a WSN [5] require the sensor network paradigm to provide mechanisms that guarantee a certain level of quality of service (QoS). Whereas the main objective in sensor network research is minimizing energy consumption, mechanisms that deliver application-level QoS efficiently and map these requirements to network-layer metrics, such as jitter and latency, have not get major concerns. Data delivery delay in WSN presents specific system design challenges, which is the object of this article.

On the hand, the joint efforts of the Zigbee Alliance [16] and the IEEE 802.15.4 [17] task group lead to the specification of a standard protocol stack for low rate wireless sensor networks , which becoming a universal solution for low cost, low power monitoring and control devices in industrial automation. Furthermore, the IEEE 802.15.4 is quite flexible for industrial DOI : $10.5121 /$ ijcnc.2011.3517 
International Journal of Computer Networks \& Communications (IJCNC) Vol.3, No.5, Sep 2011

applications if its parameters are tuned appropriately. It provides also real-time guarantees by using the Guaranteed Time Slot (GTS) mechanism [18]. However, this mechanism presents some limitations such as:

- GTS reservation is static. Slots time will be liberated only when the station that have reserved those slots time sends an explicit request for this.

- The number of the slots time is limited; so this mechanism it will be not efficient when many nodes have urgent messages.

The remainder of this article is organized as follows. Section 2 gives related works of real-time application using WSN. In section 3, we present a brief overview of the IEEE 802.15.4 protocol specifications. In section 4, we evaluate non-Beacon Mode performances for real time applications in the worst case. In section 5, we consider scenarios implemented over OMNET ${ }^{++}$ to discuss performance trends and trade-offs for real time application. In section 6 , we offer some concluding remarks.

\section{RELATED WORKS}

In [18] the authors have proposed an accurate OPNET simulation model, with focus on the implementation of the GTS mechanism. Based on the simulation model they proposed a methodology to tune the protocol parameters to guarantee better performance of the protocol by maximizing the throughput of the allocated GTS as well as minimizing frame delay.

In [19] the authors have studied the applicability of IEEE 802.15.4 based solutions in industrial automation focusing on its real-time capabilities. Their objective is to verify whether the protocol meets all the demands in industrial automation fields. Besides, they have evaluated the protocol with analytical methods focusing on its capabilities for real-time operation.

In [20] the authors have elaborated an architecture that uses the wireless protocol 802.15.4/ZigBee in a Home Automation and where it is necessary to transmit traffic flows of time-critical control data between sensors and actuators and automation network.

In [21] the authors demonstrate ZigBee's performance in several practical applications. For this purpose, authors have explained an experimental testbed setup and execution. The testbed is capable of measuring the minimum, maximum, and average received signal strength indicator (RSSI), packet loss rate (PLR), packet error rate (PER), bit error rate (BER), and the bit error locations.

In [22], the authors have presented a set of simulation experiments results to better understanding the protocol behaviour. Their results outline the capabilities and limitations of this protocol in the selected scenario. They considered the dependency of the protocol to different traffic loads and to on protocol-inherent parameters such as super-frame order and the beacon order.

In [23], the authors propose using the IEEE 802.15.4 standard with zigbee multihop routing to allow for per parking space monitoring of parking spaces. From a patron's perspective, smart parking management system can provide detailed information on the location of open parking garages and even specific parking spaces, and even allow users to reserve a parking space prior to entering the parking garages. From a parking garage manager's perspective, detailed utilization reports on individual parking spaces and overall utilization can be easily obtained, and tickets could even be automatically be issued to customers over their time limits. 
International Journal of Computer Networks \& Communications (IJCNC) Vol.3, No.5, Sep 2011

In [24] the authors showed an architecture that uses the wireless protocol 802.15.4/ZigBee in a Home Automation and where it is necessary to transmit traffic flows of time-critical control data between sensors and actuators and automation network. The propose architecture model solves the problems of limited transmission range of the IEEE 802.15.4/ZigBee protocol, allows to control and monitor the various devices in a centralized approach both locally and remotely (using the Internet access).

In [25] the authors proposed to differentiate traffic classes within the CAP, enabling lower transmission delays and higher success probability to time-critical messages, such as for event detection, GTS reservation and network management. Building upon a previously proposed methodology (TRADIF which is fully backward compatible with the IEEE 802.15.4 standard), they outline its implementation and experimental validation over a real-time operating system.

In this paper, we evaluate the IEEE 802.15.4 performance with a special focus on industrial sensor network applications.

\section{OVERVIEW OF THE IEEE 802.15.4 PROTOCOL SPECIFICATIONS}

\subsection{Description}

The 802.15.4[17] is a part of the IEEE standards family for physical and link-layers for wireless personal area networks (WPANs). The WPAN working group focuses on short-range wireless links. The main IEEE 802.15.4 focus is to provide low data rate WPANs $(0.01-250 \mathrm{Kbits} / \mathrm{s})$ with simple or no quality of service (QoS) and low complexity and rigorous power consumption requirements. The standard differentiates between full function device (FFD), and reduced function device (RFD), intended for use in the simplest of devices. The IEEE 802.15.4 protocol is being used as an enabling technology to support other protocols such as ZigBee[16] [26], 6LoWPAN [27],or WirelessHART [28][29][30] due to the great flexibility of this protocol to fit the many WSN applications requirements. A Comparison of WirelessHART and ZigBee for Industrial Applications is studied in [31].

The WirelessHART standard is specifically conceived to provide a complete solution for realtime process control applications. WirelessHART adopts IEEE 802.15.4 as the physical layer and it defines its own time-synchronized data link layer. The important features of WirelessHART data link layer include strict $10 \mathrm{~ms}$ timeslot, network-wide time synchronization, channel blacklisting, channel hopping and industry-standard AES-128 ciphers and keys. The WirelessHART network layer supports self-organizing and self-healing mesh networking techniques and uses source routing and graph routing. The particularity of WirelessHART is the use of central Network Manager which is responsible for maintaining communication schedules and up-to-date routes in order to guarantee real-time communications.

The 6LoWPAN architecture introduced an adaptation layer to trigger the development of the IPv6 over low-power WPAN. This adaptation layer works on top of physical and MAC layers, defining how IPv6 datagrams are transmitted using 802.15.4 frames by implementing compression/decompression of IPv6 headers.

The protocol stack upper layers that are defined by the ZigBee Alliance [16] [26] correspond to the Application Layer (APL) and the Network Layer (NWL), as illustrated in Figure.1.

The IEEE 802.15.4 supports two physical options. The 868/915 MHz PHY known as low-band uses binary phase shift keying (BPSK) modulation whereas the $2.4 \mathrm{GHz}$ PHY (high-band) uses Offset Quadrature Phase Shift Keying (OQPSK) modulation. The MAC layer provides sleep mode feature based on superframes bounded by the beacons. This feature is available only in the synchronized network. The ZigBee network (NWK) layer offers services for devices to join and 
leave a network, to discover and maintain routes between devices for unicast, multicast or broadcast packets and to guarantee security to data frames at network layer. The main functionalities for devices to maintain bindings, which are device groupings based upon application communication needs are provided at the ZigBee application services (APS) layer. Finally, the ZigBee application framework (AF) layer identifies a device's potential services as dictated by a given AF profile. Each ZigBee Alliance approved profile describes message formats and the network configuration parameters necessary for devices of similar interest to communicate successfully.

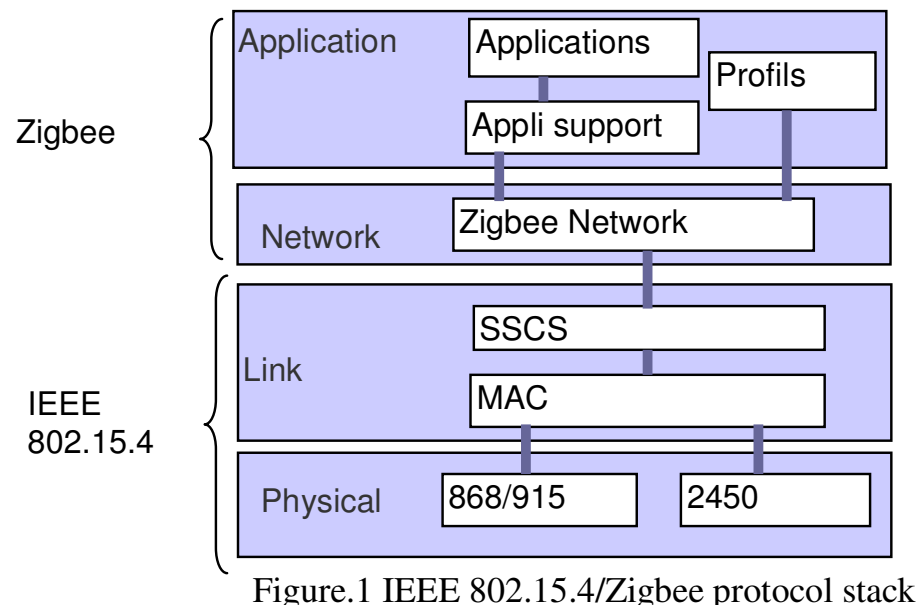

\subsection{MAC Access mechanisms \& Frame structure}

The MAC protocol in IEEE 802.15.4 can operate on both beacon enabled and non-beacon modes. In the non-beacon mode, a protocol is a simple Carrier Sense Multiple Access with Collision Avoidance (CSMA-CA). This necessitates a constant reception of possible incoming data. In the beacon-enabled mode, beacon frames are periodically sent by the PAN coordinator, to identify its PAN and synchronize nodes that are associated with it. All communications are executed in a super-frame structure. The standard allows the optional use of a super-frame structure which is sent by the coordinator bounded by network beacons. It is divided into sixteen equally sized slots. A device can transmit at any time during the slot, but must finish before the next super-frame beacon. The channel access during the time slots is contention based. For applications requiring specific data bandwidth and low latency, the network coordinator may dedicate portions of the active super-frame to that application. These portions are named guaranteed time slots (GTS). The GTS that form the contention free period (CFP), always appear at the end of the contention access period (CAP). All contention-based transactions should be achieved before the CFP begins. In a super-frame, a maximum of seven GTS slots can be allocated; involving that in each cluster (PAN) only seven nodes can have guaranteed slots in any super-frame. The remaining nodes may only transmit in the CAP, without any guarantee of quality of services parameters. The beacon-enabled mode is characterized by a Superframe Duration (SD) corresponding to the active period and a Beacon Interval (BI) specifying the time between two consecutive beacons, defined as:

$\mathrm{BI}=\mathrm{aBaseSuperframeDuration} * 2^{\mathrm{BO}}$ and $\mathrm{SD}=\mathrm{aBaseSuperframeDuration} * 2^{\mathrm{SO}}$

When $\mathrm{SO}<\mathrm{BO}$ this mean that the Beacon Interval may include an inactive period in which all nodes may enter into a sleep mode, thus saving energy.

Three additional headers are added to the outgoing data frames in the ZigBee implementations 
in order to perform the following services. In entire, ZigBee headers occupy 15 octets of overhead for each data frame. The complete IEEE 802.15.4 frame structure is represented in Figure.2. The physical packet includes many fields that correspond to the preamble (32 bits) for synchronization, the start of packet delimiter ( 8 bits) which shall be equal as "11100101", the physical header ( 8 bits) and the data field (PSDU: Payload Segment Data Unit) which has a variable length ( 0 to 1016 bits). The MAC frame structure is designed to keep the complexity at a minimum while ensuring they are sufficiently robust to be transmitted on a noisy channel. A MAC frame is composed of three fields including a MAC header (MHR), a MAC service data unit (MSDU), and a MAC footer (MFR). The frame control field (2 bytes) which is the first field of the MHR It indicates the type of the MAC frame (acknowledgement frame, data frame, beacon frame and MAC command frames), specifies format of the address field, and controls acknowledgement. The second subfield is the sequence number is used to identify successful transmission of a frame. The address field length is variable of 0 to 20 bytes. Based on the frame type, the address field may include source and destination addresses, no address or destination address. The payload (MSDU) length is variable with a restriction of 127 bytes for the complete MAC frame. The MFR corresponds to the frame check sequence (FCS) is a 16 bits used to ensure data integrity.

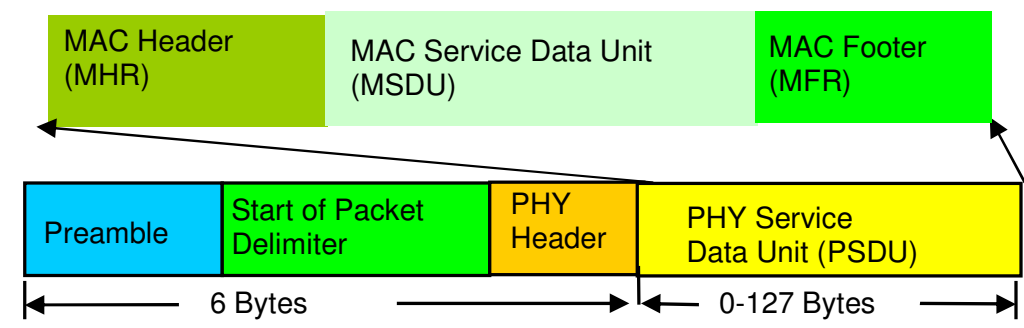

Figure.2. IEEE 802.15.4 Frame structure

\section{Beaconless Mode Performance evaluation for real time APPLICATION IN WORST CASE}

The slotted CSMA/CA backoff algorithm is based on three variables that correspond to: Backoff Exponent (BE) which used to compute the backoff delay (DB). Backoff period is the time required to transmit 20 symbols, where a symbol is equivalent to 4 bits. The number of backoff periods a device shall wait for before assessing the channel is randomly chosen between 0 and $2^{\mathrm{BE}}-1$. BE is initialized to macMinBE.

- Number of Backoffs (NB) which represents the number of times the CSMA/CA algorithm was required to backoff while attempting to access the channel. It is initialized to 0 before each new transmission attempt.

- Contention Window $(\mathrm{CW})$ that represents the number of BPs during which the channel must be sensed idle before channel access. It is used only in the slotted mode.

The IEEE 802.15.4 CSMA-CA algorithms realize energy savings by keeping the node idle during the backoff procedure. A node executes the Clear Channel Assessment (CCA) procedure. The most significant parameters in the unslotted CSMA/CA are "macMaxFrameRetries" $(0 \leq$ Maximum number of retransmissions $\leq 7)$, "macMaxCSMABackoff" $(0 \leq$ Maximum number of backoff stages $\leq 5)$, "macMaxBE" $(3 \leq \operatorname{macMaxBE} \leq 8)$ and "macMinBE" $(0 \leq \operatorname{macMinBE} \leq$ 7). The unslotted algorithm is illustrated in Figure 3. 


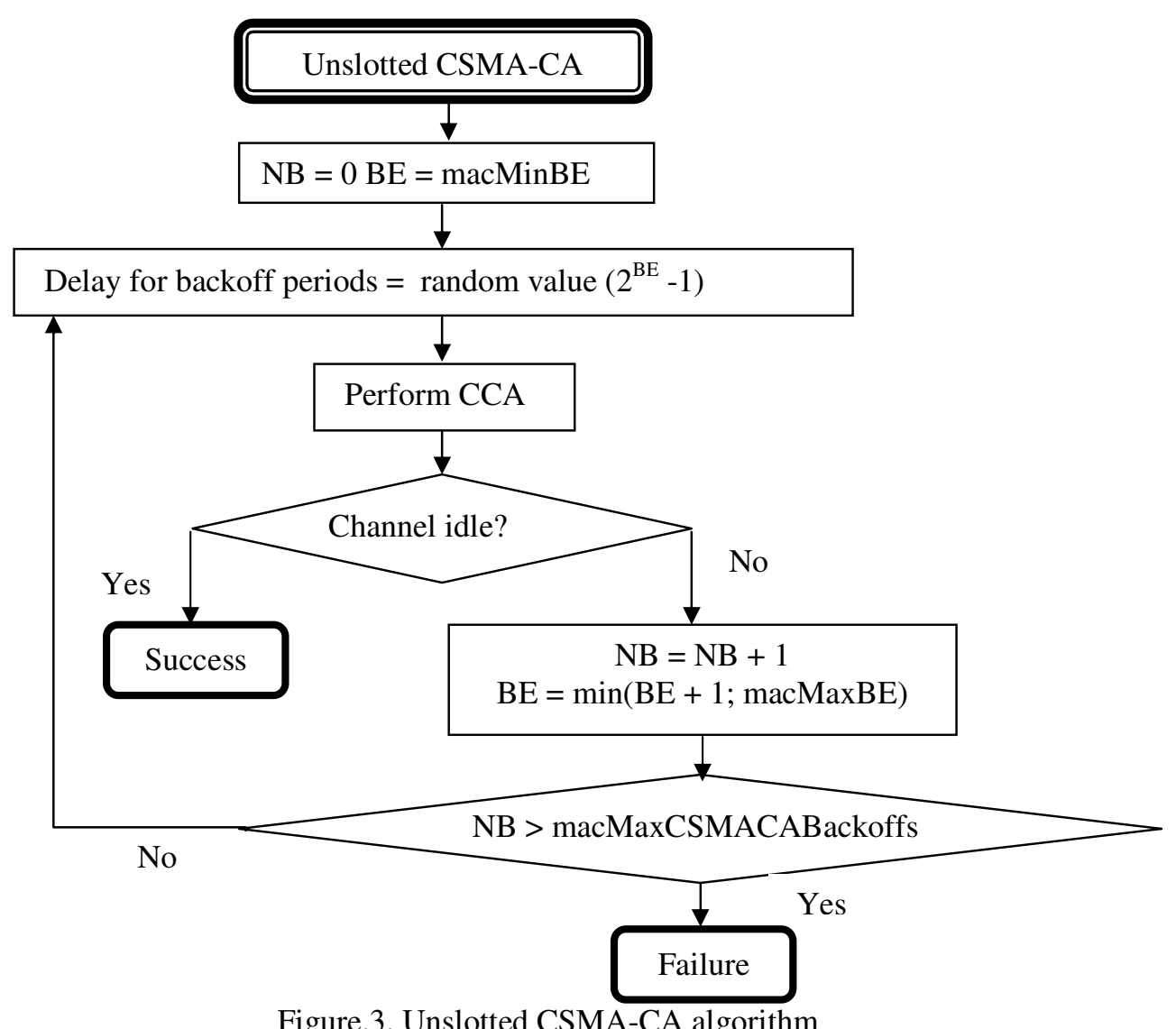

In order to know the impact of the Unslotted CSMA-CA algorithm we compute the End-to-End delay in the worst case this mean that the unslotted CSMA/CA parameters taking their maximum values $($ macMaxFrameRetries $=7 ;$ macMaxCSMABackoff $=5 ;$ macMaxBE $=8)$;

$\mathrm{E}_{2} \mathrm{E}_{\mathrm{WC}}=$ macMaxFrameRetries $*\left(\mathrm{D}_{\mathrm{BOWC}}+\right.$ Tframe $\left.+\mathrm{T}_{\tau \mathrm{A}}+\mathrm{T}_{\text {ack }}+\mathrm{T}_{\mathrm{LIFS}}\right)$

Eq.1

The following notations were used:

$\mathrm{E} 2 \mathrm{E}_{\mathrm{WC}}=$ End-to-End delay in the Worst case;

$\mathrm{D}_{\text {BowC }}=$ Back off period in the worst case in seconds,

Tframe $=$ Transmission time for a frame with maximum payload length

$\mathrm{T}_{\tau \mathrm{A}}=$ Turn around time: time between data frame and its corresponding ACK;

$T_{a c k}=$ Transmission time for an ACK

$\mathrm{T}_{\mathrm{LIFS}}=\mathrm{IFS}$ time For the IFS, LIFS is used when the MPDU is greater than 18 bytes.

In the following, we will calculate the different times.

- The Worst case back off period is expressed as follows:

$\mathrm{D}_{\text {BowC }}=$ macMaxCSMABackoff $*\left(\mathrm{~N}_{\mathrm{BO}} * \mathrm{~T}_{\text {BOslot }}\right)$;

Were $\mathrm{N}_{\mathrm{BO}}=$ maximum Number of back off slots and $\mathrm{T}_{\text {slot }}=$ Time for a back off slot.

Then $\mathrm{N}_{\mathrm{BO}}=2^{\text {macMaxBE }}-1=2^{8}-1=255$;

$\mathrm{T}_{\text {BOslot }}=20 \mathrm{~T}_{\text {symbol }}=20 * 4 * 1 /$ (Bit rate) ;

The Bit rate depends on the frequency bands; the smaller value is $20 \mathrm{Kbps}(868.0-868.6 \mathrm{MHz}$; modulation scheme $=$ BPSK). Therefore $\mathrm{T}_{\text {BOslot }}=3.90625 \mathrm{~ms} ; \mathrm{D}_{\text {BоwC }}=4.98046875 \mathrm{~s}$.

- $\quad$ Trame $=(133 * 8) /(20 * 1024)=51.953125 \mathrm{~ms}$.

- $\quad \mathrm{T}_{\tau \mathrm{A}}=12 * \mathrm{~T}_{\text {symbol }}=12 * 4 * 1 /($ Bit rate $)=12 * 4 / 20 * 1024=2.34375 \mathrm{~ms}$

- $\quad T_{a c k}=(31 * 8) /(20 * 1024)=12.109375 \mathrm{~ms}$

- $\quad \mathrm{T}_{\mathrm{LIFS}}=40 * \mathrm{~T}_{\text {symbol }}=7.8125 \mathrm{~ms}$ 
International Journal of Computer Networks \& Communications (IJCNC) Vol.3, No.5, Sep 2011

$\mathrm{E} 2 \mathrm{E}_{\mathrm{WC}}=7 *\left(4.98046875+(51.953125+2.34375+12.109375+7.8125) * 10^{-3}\right)=35.3828125 \mathrm{~s}$ In the worst case and when the Unslotted CSMA-CA algorithm is used the $\mathrm{E}_{2} \mathrm{E}_{\mathrm{WC}}$ is equal to $35.38 \mathrm{~s}$. Therefore, under unsaturated networks condition, Unslotted CSMA-CA can be used in real time application.

\section{IEEE 802.15.4 PERFORMANCE ANALYSIS FOR REAL TIME APPLICATION IN NON-BEACON MODE}

In this section, we are interested in wireless sensors networks performances analysis for real times applications in non-beacon mode. This mode has the least overhead so it will give us an upper bound on the maximum throughput of the protocol. The performance evaluation is performed using a simulation model of IEEE 802.15.4 under OMNeT++ [32][33]. In this section, we introduced this model and we described the simulation settings.

\subsection{Simulations modules}

The principles modules constituting the IEEE 802.15.4 network (shown in Figure.4) are the Sim module, the host module and the Nic802154_TI_CC2420 module.

- Host Module is the node that sends and receives the messages.

- BlackBoard is an entity that allows the communication inters layer/process. It builds the total knowledge of the hosts or relevant information for more than one layer accessible for modules derived from BlackBoard Acces. Each module derived from BlackBoardAcces is able to publish information in BlackBoard.

- SimTracer allows to record vectors in files .vec (result of simulation).

- ChannelControl is a framework module that allows controlling connections. It coordinates connections between all the nodes.

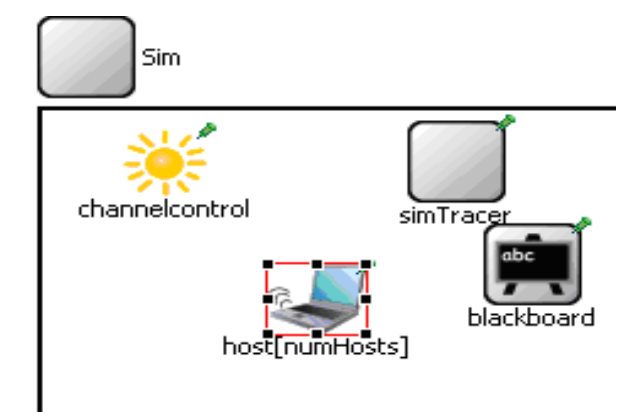

Figure.4 IEEE802.15.4 network.ned module

The Host module it contains four simple modules and a compound module. Those modules are SensorApplayer, WiseRoute, UbiquitousArp and Nic802154_TI_CC2420. Figure.5 illustrates the host module.

- SensorApplayer it implements the higher layers

- WiseRouteis the basic class for the network layer; it is a generic class for all modules of network layer.

- ConstSpeedMobility it controls all the nodes movements. UbiquitousArpis a module that implement address resolution protocol.

- Nic802154_TI_CC2420 module is a compound module and the structure of this module is illustrated below by Figure.6. 


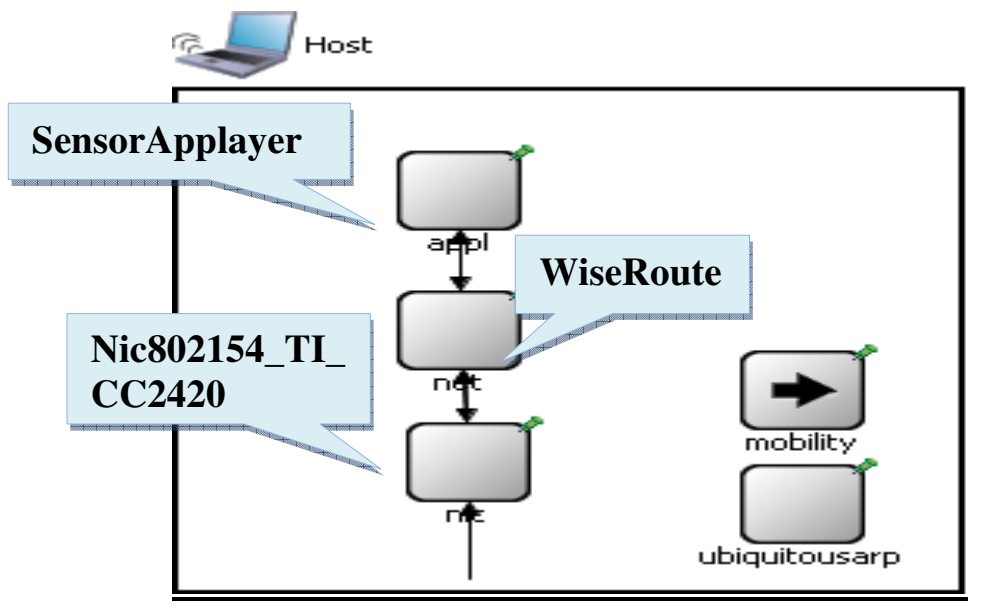

Figure.5 IEEE802.15.4 Host.ned module

Nic802154_TI_CC2420 module contains 4 simple modules:

- MAC module is based on CSMA/CA algorithm.

- SnrEval can be used in order to consider some information for the received messages since the module.

- SnrDecider processes the received data to verify the message integrity then to decide if the message can be dropped. In order to take decision the Snr decider takes the snrList created by snrEval and translates SNR values to the bit state (with or without error).

- SingleChannelRadioAccNoise3 contains the Nic802154_TI_CC2420 parameters.

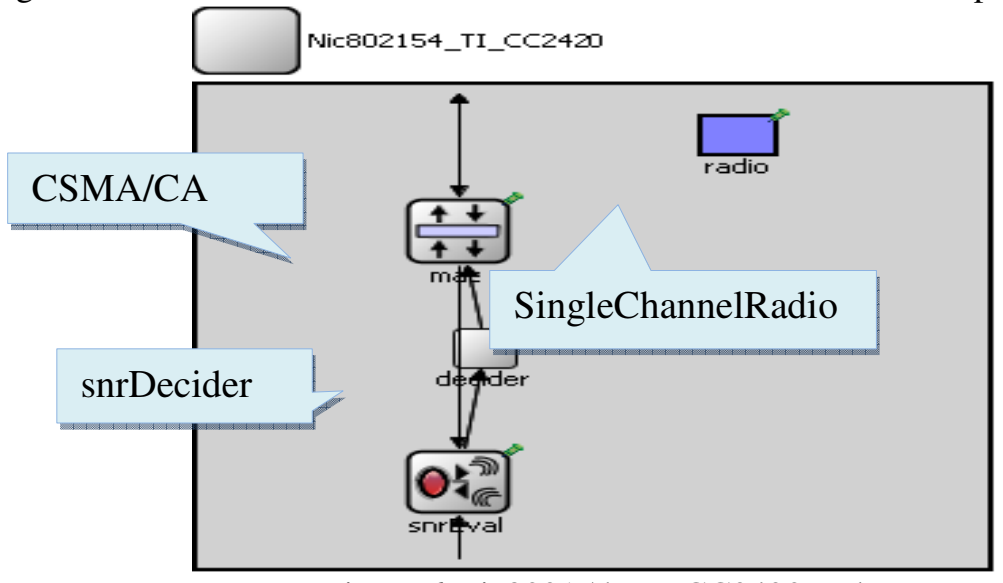

Figure.6 Nic802154_TI_CC2420.ned

\subsection{Simulation parameters}

We have evaluated the 802.15.4 protocol performances in star topology, where nodes transmit data directly to the coordinator. We considered that the wireless channel is without interferences, so this means that each node is able to hear the transmissions of all the other nodes. The CSMA/CA mechanism uses imply that the messages cannot be delivered because of collisions or impossibility of reaching an occupied channel.

In this scenario, every $30 \mathrm{~ms}$, the sensors generate messages of 108 bytes. These messages are composed of 75 bytes of data, 2 bytes of control added by the request, the ZigBee heading is equal to 20 bytes, and 9 bytes corresponds to the heading included by the 802.15 .4 protocol. The delivery acknowledgement (ACK) sent by the coordinator is composed of 5 bytes. The 
Sensors start to generate the data at random moment between the beginning of simulation and $30 \mathrm{~ms}$. Each sensor sends 1000 messages to the coordinator. In the table one and table two, we have regrouped respectively the CSMA/CA and the Nic802154_TI_CC2420 parameters used during simulation.

Table.1 CSMA/CA Parameter

\begin{tabular}{|l|l|}
\hline Parameter & Value \\
\hline minBE & 3 \\
\hline maxBE & 5 \\
\hline MaxCSMABackoffs & 4 \\
\hline MaxFrameRetries & 3 \\
\hline AckWaitDuration & $864 \mu \mathrm{s}$ \\
\hline SIFS & $192 \mu \mathrm{s}$ \\
\hline AUnitBackoffPeriod & $320 \mu \mathrm{s}$ \\
\hline CCADetectionTime & $128 \mu \mathrm{s}$ \\
\hline
\end{tabular}

Table.2 Nic802154_TI_CC2420 Parameter

\begin{tabular}{|l|l|}
\hline Parameter & Value \\
\hline DelaySetupRx & $1.792 \mathrm{~ms}$ \\
\hline DelaySetupTx & $1.792 \mathrm{~ms}$ \\
\hline delayRxTx / delayTxRx & $192 \mu \mathrm{s}$ \\
\hline
\end{tabular}

Several parameters can be defined to evaluate the wireless network performances. These measurements were carefully selected to give an idea of the ZigBee networks behaviour and reliability. The considered parameters are as follows:

- Throughputs is the data quantity transmitted correctly starting from the source to the destination within a time $\mathrm{U}(\mathrm{sec})$. The node rate is measured by counting the total number of data packets received successfully on the node, which leads to the calculation of the received bits number which is divided by the total time of simulation execution. The network rate is defined as the average of all nodes rates implied in the data transmission.

- End-to-End-delay End-to-End Delay: it is a crucial metric to evaluate the real-time performance of networks. is equal to the time taken by a data packet to reach the destination node. Simulations are carried out with a star network topology, and the routing mechanism was desactivated. Consequently, the term End-to-End Delay can be exchanged with delay. The packet delay is the time put by the packet to reach the destination. Therefore, it is calculated as the average time difference between the points when a packet is generated at the network device (transmitter) and when the packet is received by the network coordinator (receiver).

- Goodput is calculated as the rate between packets received correctly and the total transmitted packets. The lost packets number does not take into account the retransmissions.

- Latency time is equal to the arrived message time minus the message creation time.

- Service time is the message life time from its creation by the transmitter until the positive ACK reception.

\section{Scenarios}

\subsubsection{Scenario1}

In this scenario (star topology one coordinator and $\mathrm{n}$ nodes), in first step, we have fixed the sensors number, then for different rates we have computed the goodput and the latency. In the second step, we change the number of sensors and we repeat the same process. Figure. 5 illustrates simulations results corresponding to this scenario. 
International Journal of Computer Networks \& Communications (IJCNC) Vol.3, No.5, Sep 2011

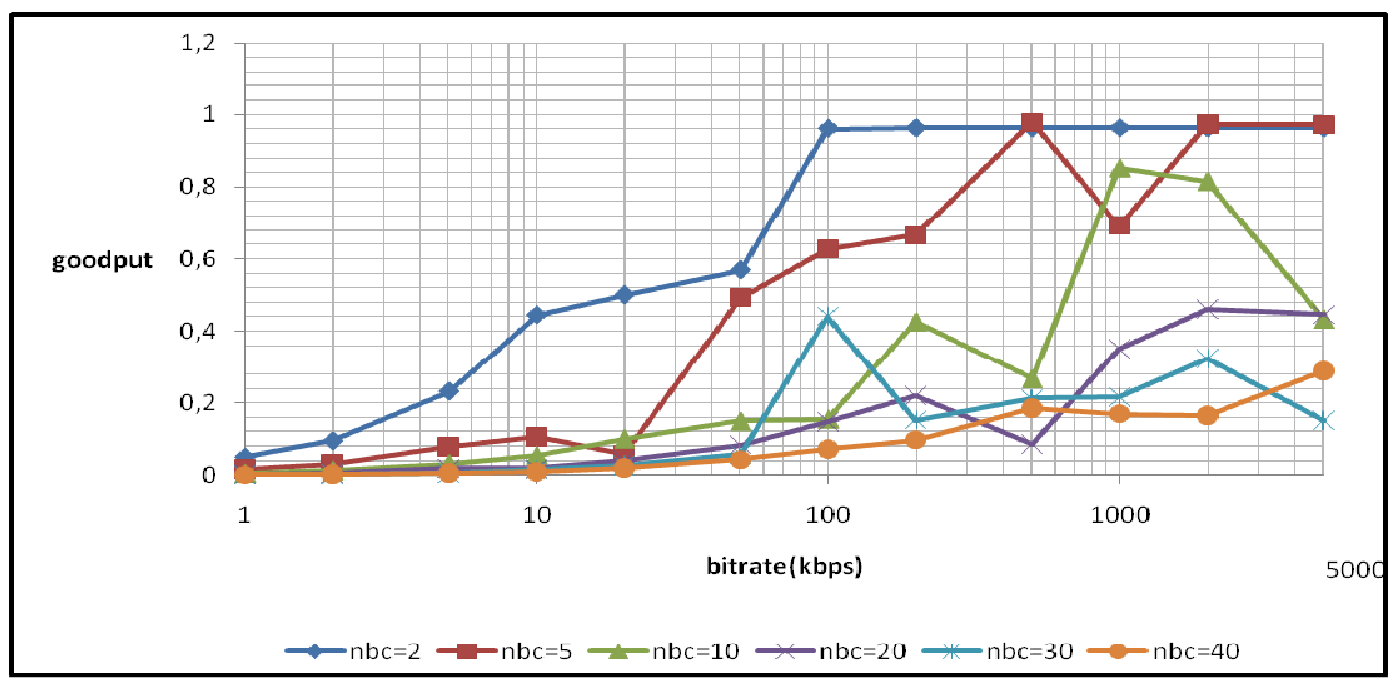

Fig.6 Goodput according to the rate for various numbers of sensors

For the curve $(n b c=2)$ we notice that the goodput reached a threshold. The rate increases doesn't have any advantages. For the curves $(n b c=5$ and $n b c=10)$ we perceive that the goodput converge to the ideal value when the rate increases. For the curves $(n b c=20, n b c=30$ and $n b c=40$ ), the goodput has a low value even though the rate increases. According to the network size, If the number of sensor is high, it is necessary to have a high rate to ensure a correct network operation.

For the curves illustrated by Figure.7 which represent the goodput according to the simulation time we have fixed the rate equal to $250 \mathrm{Kbps}$.

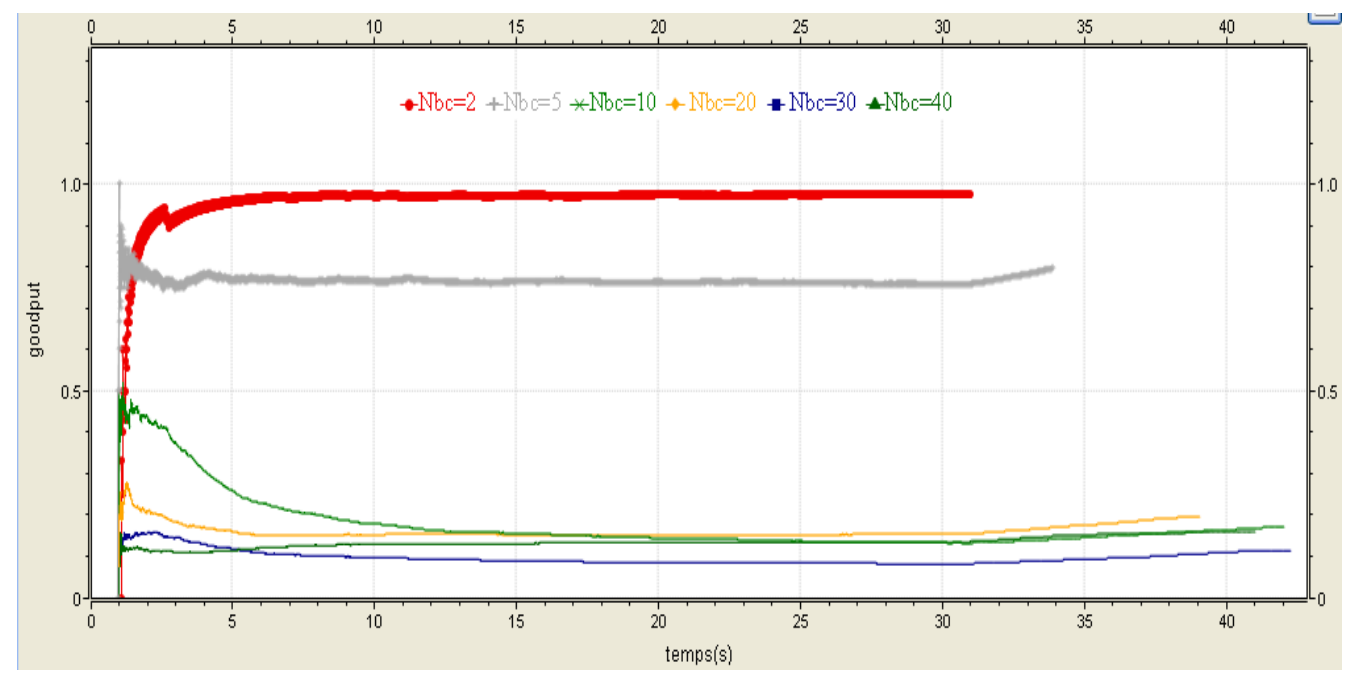

Figure.7 Goodput according to the simulation time

The latency time variation according to the rate for various numbers of sensors is illustrated by Figure.8. 


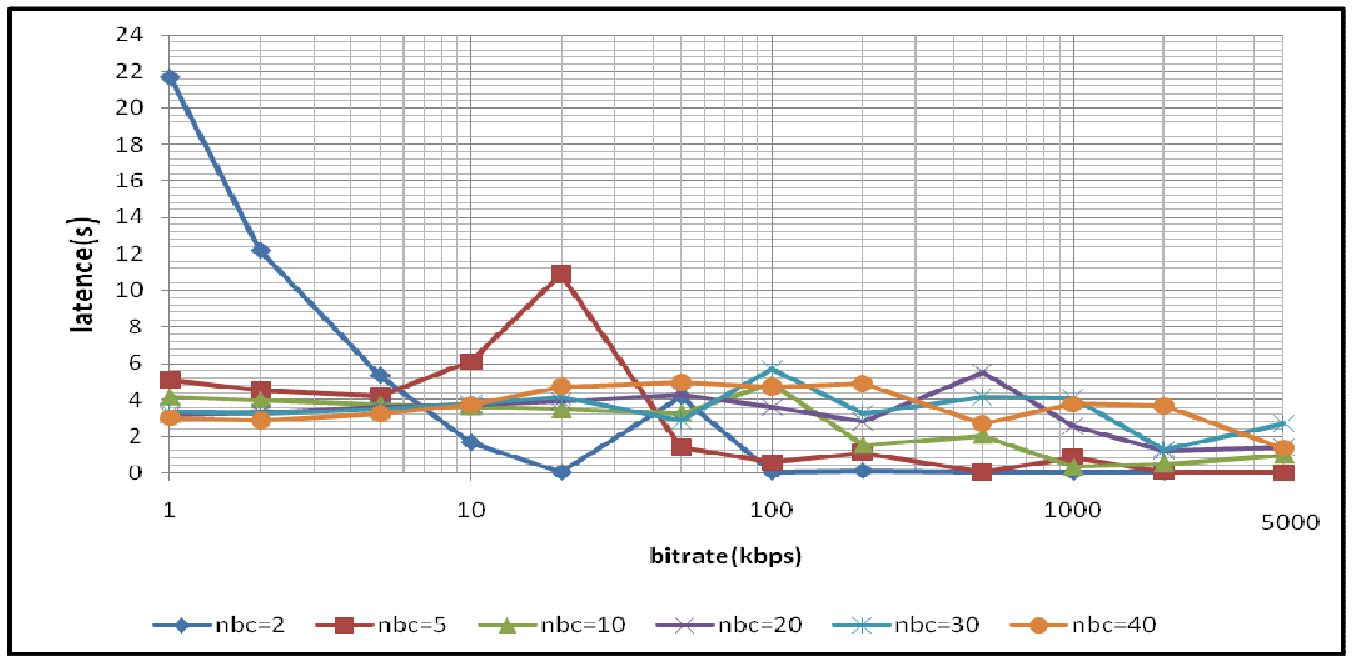

Figure.8 Latency according to the rate for various numbers of sensors

For the curve (nbc=2, rate $100 \mathrm{Kbps}$ ) the latency time is limited by $15 \mathrm{~ms}$. For the curves $(n b c=5$ and $n b c=10)$ the latency time varies according to the rate. For the curves $(n b c=20$, $n b c=30$ and $n b c=40$ ) the rate does not have a great effect on the latency time. When the number of sensor is increasing the rate doesn't guaranteed the latency time decreases because of the collisions and the messages losses.

In Figure. 9 we illustrates the latency time variation according to simulation time for various rate values (sensors number $=10$, packet number $=100$ ).

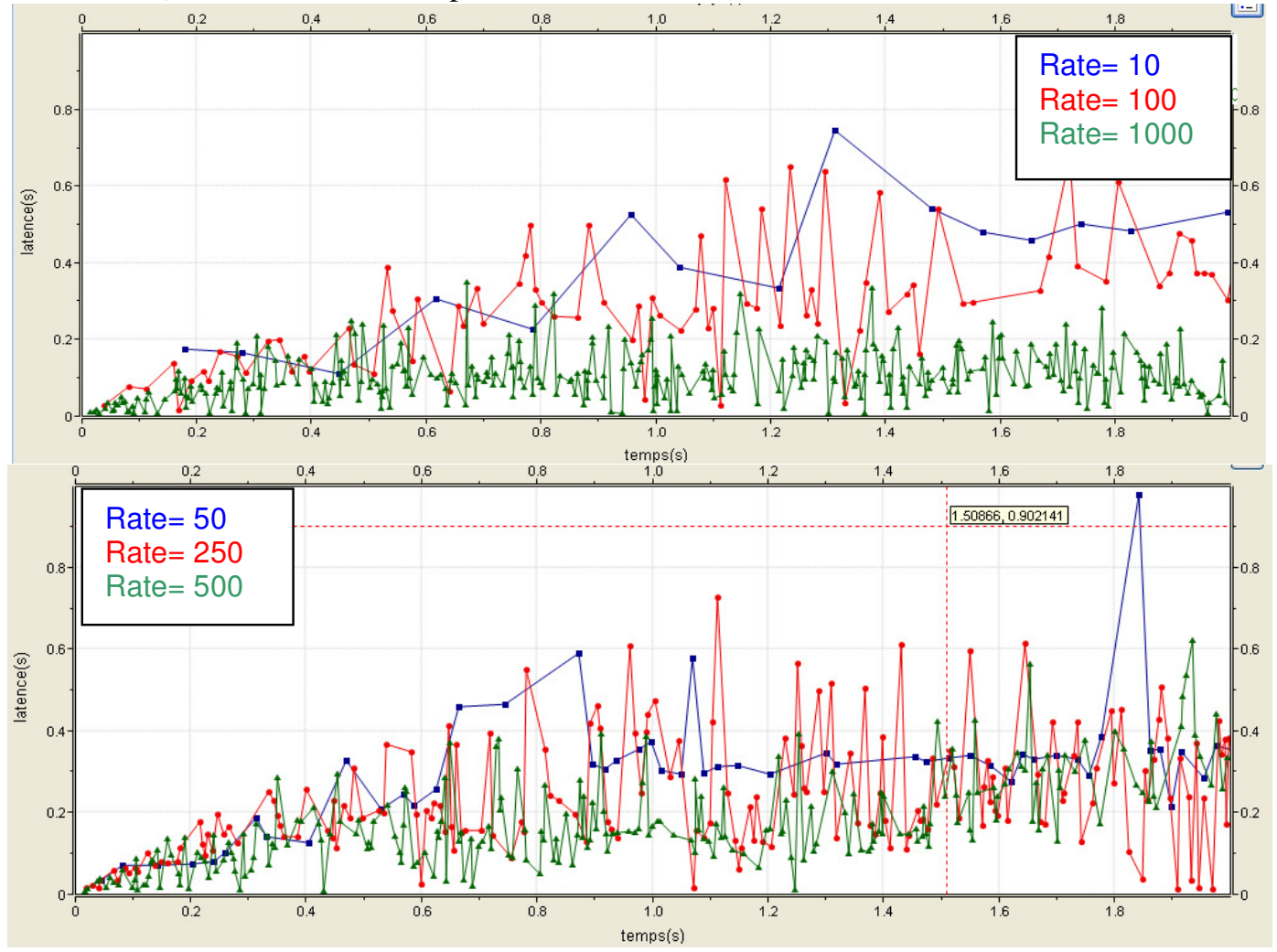

Figure.9 Latency time according to the simulation time 
International Journal of Computer Networks \& Communications (IJCNC) Vol.3, No.5, Sep 2011

In order to evaluate the service time variation according to different rate as shown in Figure.10, we have considered that every one second the sensors generate messages; each sensor sends 100 messages to the coordinator. We have fixed the number of sensor equal to five.
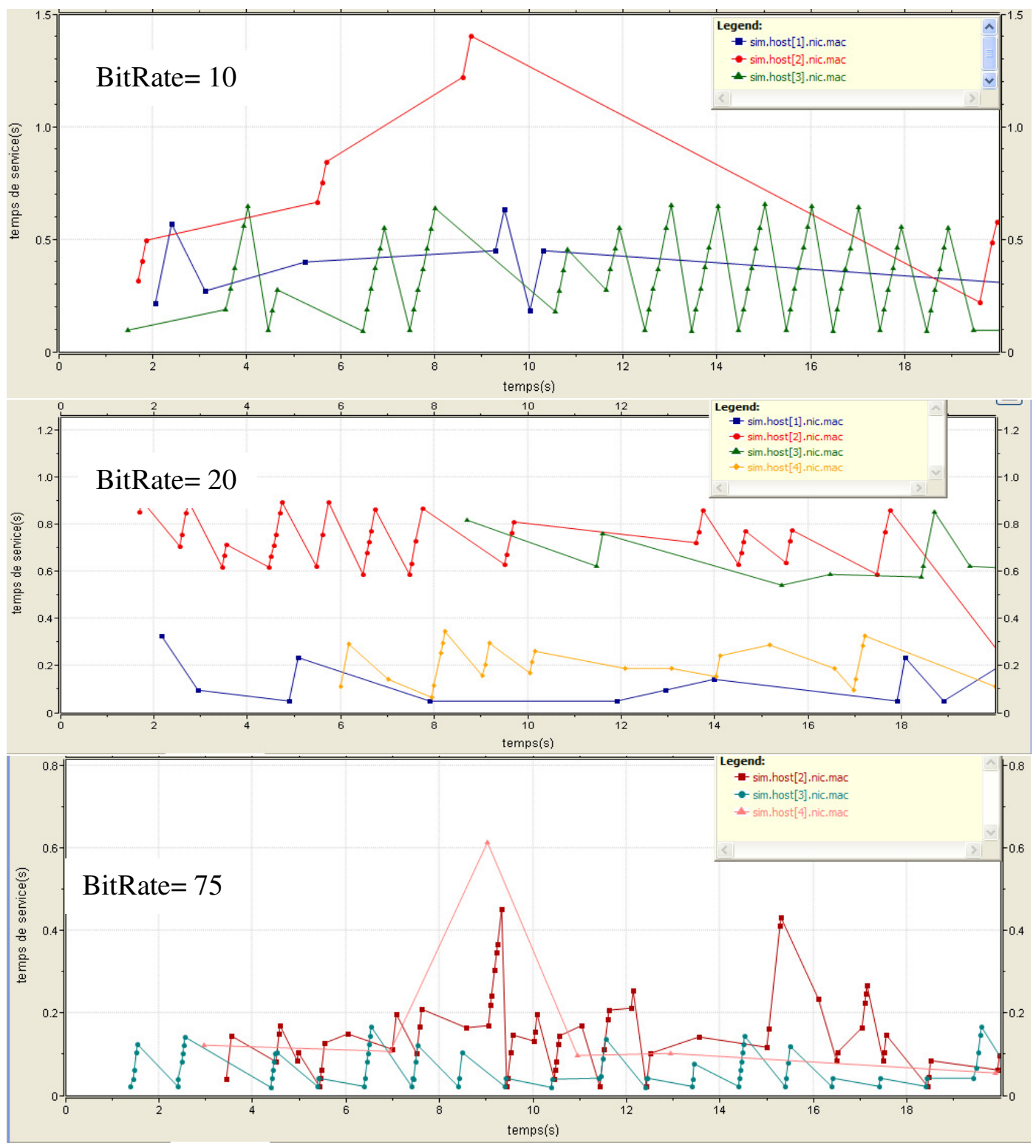

Figure.10 Service time according to the simulation time

\subsubsection{Scenario 2: Payload size analysis}

In this scenario, we have analyzed the IEEE 802.15.4/ZigBee performances using a star topology for different payload sizes and ten sensors. We seek to determine the performances according to the rate in order to estimate the suitable payload size for a given rate. We have used the network parameters of the preceding scenario. Simulations results, illustrated respectively by figures 11,12 and 13 . 
International Journal of Computer Networks \& Communications (IJCNC) Vol.3, No.5, Sep 2011

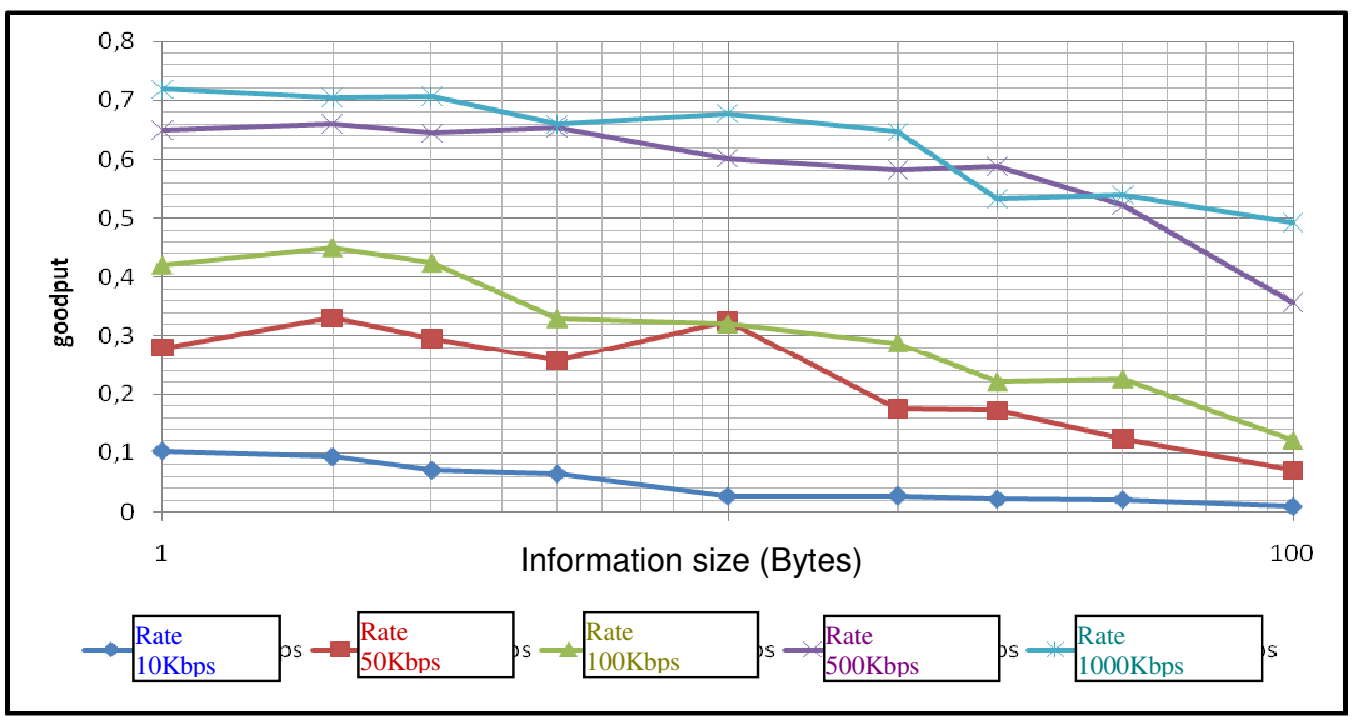

Figure.11 Goodput variation according to payload information size

According to the obtained results, there isn't correlation between the latency and the useful information size in the real times applications case (when the information size doesn't exceed 100 bytes).

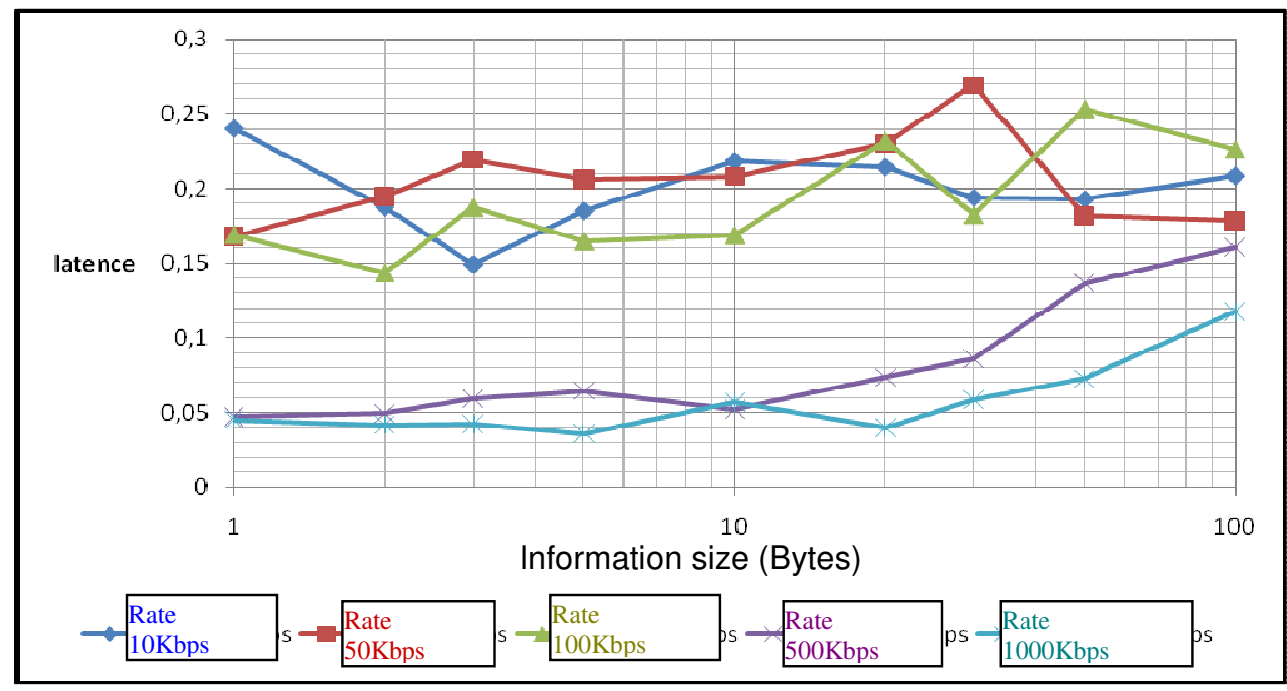

Figure.12 Latency variation according to payload size

In order to evaluate the service time variation according to different rate as shown in Figure.13, we have considered that every one second the sensors generate messages with $20 \mathrm{Kbps}$. We have fixed the sensor number at 5 . 
International Journal of Computer Networks \& Communications (IJCNC) Vol.3, No.5, Sep 2011
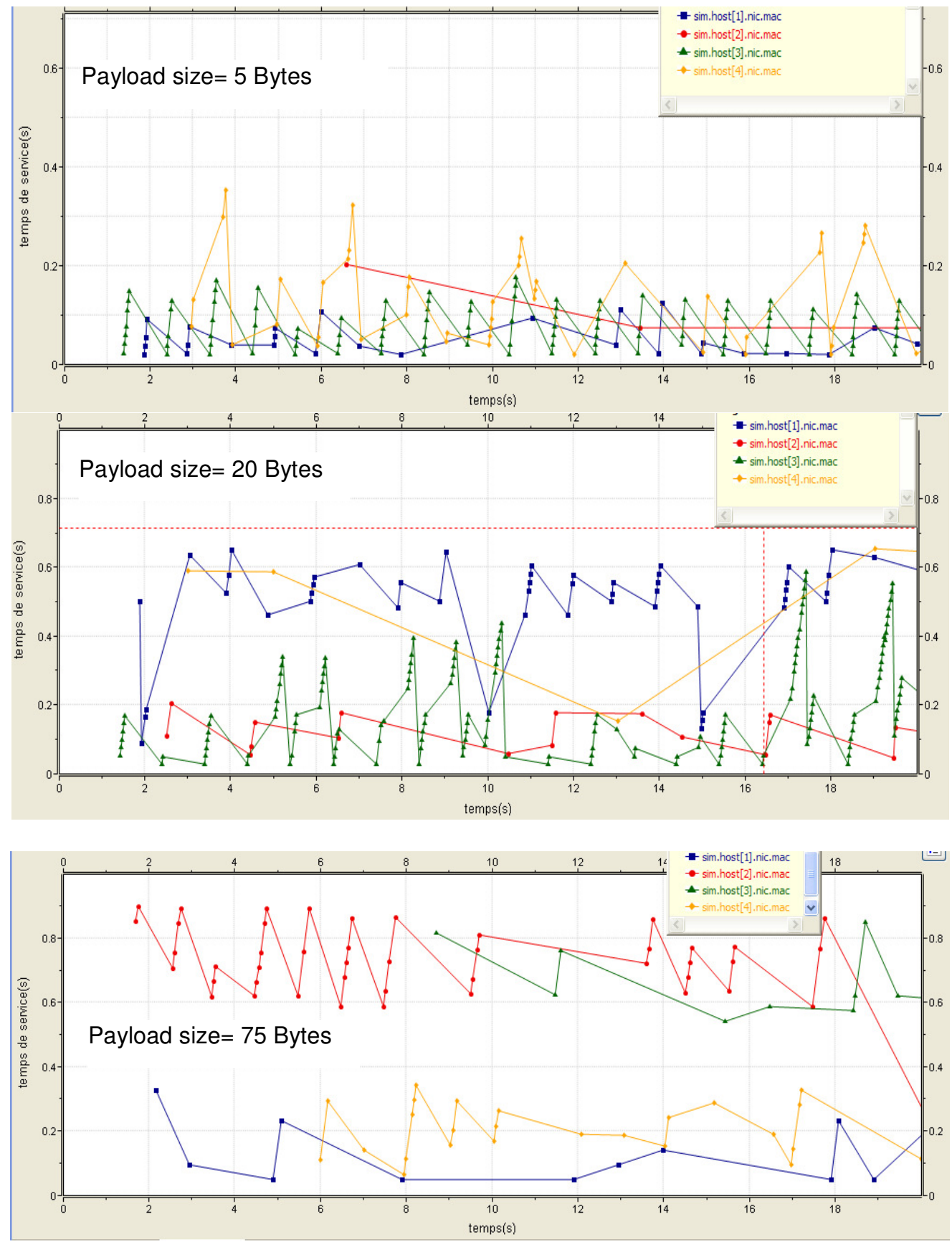

Fig.13 Service time variation according to payload size

\section{Conclusion}

In this paper, we have presented and discussed a number of performance measures of the IEEE 802.15.4 protocol. In essence, we obtained these results in a simulative performance study using IEEE 802.15.4 simulation model, which is implemented under OMNeT++. We have analyzed two different scenarios for the CSMA/CA operation mode using a star topology. In the first scenario, we treated the scalability problem by evaluating the metric performance 
International Journal of Computer Networks \& Communications (IJCNC) Vol.3, No.5, Sep 2011

(goodput, latency) for different network sizes (different sensors number). In addition, in the second scenario, we analysed payload sizes effect on the IEEE 802.15.4/ZigBee performances. In both scenarios, we have considered non beacon mode. In future work, we will continue to study the applicability of IEEE 802.15.4 in low-latency and energy-aware applications especially in real network and for healthcare use.

\section{REFERENCES}

[1] I. F. Akyildiz, W. Su, Y. Sankarasubramaniam, and E. Cayirci. Wireless sensor networks: a survey. Elsevier Computer Networks, 38:393-422, 2 I002.

[2] P. Baronti, P. Pillai, V. W. Chook, S. Chessa, A. Gotta, and Y. F. Hu. Wireless Sensor Networks: a Survey on the State of the Art and the 802.15.4 and ZigBee Standards. Elsevier Computer Communications, 30(7):1655-1695, May 2007.

[3] KAZEM SOHRABY, DANIEL MINOLI, TAIEB ZNATI “WIRELESS SENSOR NETWORKS Technology, Protocols, and Applications, 2007 by John Wiley \& Sons, Inc, ISBN 978-0-47174300-2.

[4] Tien-Wen Sung, Ting-Ting Wu, Chu-Sing Yang, Yueh-Min Huang, "RELIABLE DATA BROADCAST FOR ZIGBEE WIRELESS SENSOR NETWORKS", INTERNATIONAL JOURNAL ON SMART SENSING AND INTELLIGENT SYSTEMS, VOL. 3, NO. 3, SEPTEMBER 2010

[5] Paolo Pagano, Mangesh Chitnis,Giuseppe Lipari, Christian Nastasi, and Yao Liang, "Simulating Real-Time Aspects of Wireless Sensor Networks", Research Article doi:10.1155/2010/107946, EURASIP Journal on Wireless Communications and Networking Volume 2010 (2010), Article ID 107946, 19 pages

[6] Ang, Won-Suk, William M.Healy, "Assessment of Performance Metrics for Use of WSNs in Buildings", 2009 International Symposium on AUTOMATION and ROBOTICS in CONSTRUCTION (ISARC 2 009) p.570-575, 2009

[7] Hussein T. Mouftah, Mounib Khanafer and Mouhcine Guennoun, “Wireless Sensor Network Architecturesfor Intelligent Vehicular Systems, Symposium international for telecommunication techniques, 2010

[8] Changsu Suh a, Zeeshan Hameed Mir b, Young-Bae Ko, “ Design and implementation of enhanced IEEE 802.15.4 for supporting multimedia service in Wireless Sensor Networks", Computer Networks 52 (2008) 2568-2581, Elsevier

[9] N. GolmieD. CypherO. Rebala "Performance analysis of low rate wireless technologies for medical applications", Computer Communications Volume 28 , Issue 10 June 2005 Pages: 1266-1275 ISSN:0140-3664.

[10] Huiling Zhoul, Xuechuan Chen, Xiangdong Liu and Jun Yang, "Applications of Zigbee Wireless Technology Tomeasurement System in Grain Storage", Computer and Computing Technologies in Agriculture II, Volume 3, IFIP International Federation for Information Processing, 2009, Volume, 2021-2029, DOI: 10.1007/978-1-4419-0213-9_52

[11] Pedro Mestre, , Carlos Serôdio, Raul Morais, Jorge Azevedo, Pedro Melo-Pinto, "Vegetation Growth Detection Using Wireless Sensor Networks", Proceedings of the World Congress on Engineering 2010 Vol I WCE 2010, June 30 - July 2, 2010, London, U.K.

[12] A. Willig, "Recent and emerging topics in wireless industrial communication," IEEE Transactions on Industrial Informatics, vol. 4, no. 2, pp. 102-124, 2008.

[13] Pangun Park, "Protocol Design for Control Applications using Wireless Sensor Networks", Thesis, Automatic Control Lab School of Electrical Engineering, KTH (Royal Institute of Technology), Stockholm, Sweden, 2009, ISSN 1653-5146, ISBN 978-91-7415-441-5 
International Journal of Computer Networks \& Communications (IJCNC) Vol.3, No.5, Sep 2011

[14] F. Chen, T. Talanis, R. German, and F. Dressler, "Realtime Enabled IEEE 802.15.4 Sensor Networks in Industrial Automation," in IEEE Symposium on Industrial Embedded Systems (SIES 2009). Lausanne, Switzerland: IEEE, July 2009, pp. 136-139.

[15] Marcel Meli, Martin Gysel Marc Sommerhalder "Using IEEE 802.15.4 / ZigBee in audio applications", Embedded World, Nuremberg, February 2006

[16] ZigBee Alliance (2005), “ZigBee specification”, 2004. Available at http://www.zigbee.org.Zigbee

[17] IEEE 802.15.4-2006 Standard for Information technology- Telecommunications and information exchange between systems- Local and metropolitan area networks- Specific requirements Part 15.4: Wireless Medium Access Control (MAC) and Physical Layer (PHY) Specifications for Low-Rate Wireless Personal Area Networks (WPANs). IEEE Computer Society. Revision of IEEE Std 802.15.4-2003). ISBN 0-7381-4996-9. 8 September 2006.

[18] Petr Jurčík, Anis Koubâa, Mário Alves, Eduardo Tovar, Zdeněk Hanzále, "A Simulation Model for the IEEE 802.15.4 Protocol: Delay/Throughput Evaluation of the GTS Mechanism”, 15th International Symposium on Modeling, Analysis, and Simulation of Computer and Telecommunication Systems, 2007. MASCOTS '07, 24-26 Oct. 2007, page(s): 109 - 116 , Istanbul, ISSN: 1526-7539, Print ISBN: 978-1-4244-1853-4.

[19] Feng Chen Talanis, T. German, R. Dressler, F. "Real-time enabled IEEE 802.15.4 sensor networks in industrial automation", IEEE International Symposium Industrial Embedded Systems, 2009. SIES '09. ISBN: 978-1-42444109-9, INSPEC Accession Number: 1081428

[20] Collotta M., Salerno V. M. “A real-time network based on IEEE 802.15.4 / ZigBee to control home automation environment", International forum "Modern Information Society Formation problems, perspectives, innovation approaches". St.-Petersburg, Russia, June 6-11, 2010

[21] Patrick R. Casey, Kemal E. Tepe, and Narayan Kar, “ Design and Implementation of a Testbed for IEEE 802.15.4 (Zigbee) Performance Measurements”, Research Article, EURASIP Journal onWireless Communications and Networking, Volume 2010, Article ID 103406, 11 pages, doi:10.1155/2010/103406, Hindawi Publishing Corporation.

[22] Feng Chen, Nan Wang, Reinhard German and Falko Dressler, "Simulation study of IEEE 802.15.4 LR-WPAN for industrial applications", Wirel. Commun. Mob. Comput. 10: 609-621 (2010) Published online in Wiley InterScience (www.interscience.wiley.com) DOI: 10.1002/wcm.736

[23] D Rohm, M Goyal, N Dua, H Hosseini, K Vairavan, "Smart Parking Lots of the Future", Symposium (RTAS) 2007: Workshop on Smart Transportation, April 3, 2007, Bellevue, WA

[24] Mario Collotta, Valerio Mario Salerno, “A REAL-TIME NETWORK BASED ON IEEE 802.15.4/ZIGBEE TO CONTROL HOME AUTOMATION ENVIRONMENT”, ISA European paper competition - Modern Information society formation - Problems Perspectives, innovation Approaches, ISBN/ISSN: ISBN 978-5-8088-0528-6 - 6-11 June 2010.

[25] Ricardo Severino, Manish Batsa, Mário Alves, Anis Koubâa, "A Traffic Differentiation Add-On to the IEEE 802.15.4 Protocol: implementation and experimental validation over a real-time operating system", 13th Euromicro Conference on Digital System Design, Architectures, Methods and Tools, DSD 2010, 1-3 September 2010, Lille, France. IEEE 2010, ISBN 978-07695-4171-6

[26] Anneleen Van Nieuwenhuyse, Mário Alves, Anis Koubâa, "On the use of the ZigBee protocol for Wireless Sensor Networks”, HURRAY-TR-060603, 26/JUN/2006.

[27] 6LoWPAN Working Group, "IPv6 over Low power WPAN", http://www.ietf.org/dyn/wg/charter/6lowpan-charter.html, 2010.

[28] HCF - HART Communication Foundation, "HART7 Specification”, September 2007.

[29] Jianping Song, Song Han, Aloysius K. Mok, Deji Chen, Mike Lucas, Mark Nixon, "WirelessHART: Applying Wireless Technology in Real-Time Industrial Process Control", IEEE Real-Time and Embedded Technology and Applications Symposium, (2008), 377-386. 
International Journal of Computer Networks \& Communications (IJCNC) Vol.3, No.5, Sep 2011

[30] Song Han, Xiuming Zhu, Aloysius K. Mok, Deji Chen, Mark Nixon, “ Reliable and Real-time Communication in Industrial Wireless Mesh Networks", 17th IEEE RealTime and Embedded Technology and Applications Symposium (RTAS), Chicago, IL, 2011.

[31] Lennvall, T.; Svensson, S.; Hekland, F.; "A comparison of WirelessHART and ZigBee for industrial applications", Factory Communication Systems, 2008. WFCS 2008. IEEE

International Workshop on , 21-23 May 2008, pp 85 - 88, Dresden , Print ISBN: 978-1-42442349-1

[32] Http://www.omnetpp.org/

[33] Feng Chen, OMNeT++ IEEE 802.15.4 Model for OMNeT++/INET Framework Available http://www7.informatik.uni erlangen.de/ fengchen/omnet/802154/.

\section{Authors}

Dr Lamia CHAARI was born in Sfax, Tunisia, in 1972. She received the engineering and $\mathrm{PhD}$ degrees in electrical and electronic engineering from Sfax national engineering school (ENIS) in TUNISIA. Actually she is an assistant professor in multimedia and informatics higher institute in SFAX She is also a researcher in electronic and technology information laboratory (LETI). Her scope of research are communications, networking and signal processing which are specially related to wireless and new generation networks.

Prof Lotfi Kamoun was born in Sfax Tunisia, 25 January. 1957. He received the electrical engineering degree from the Sciences and Techniques Faculty in Tunisia. Actually he is a Professor in Sfax national engineering school (ENIS) in TUNISIA, Director of SFAX Higher Institute of Electronics and Communications in Tunisia and director of electronic and technology information laboratory (LETI). His scope of research are communications, networking, and Software radio and signal processing which are specially related to wireless and new generation

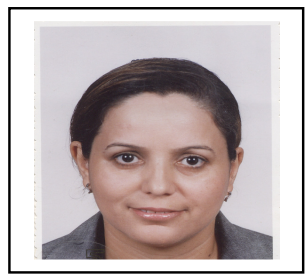
networks.

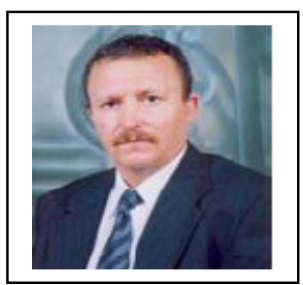

